\title{
A CONTROLLED CURRENT INVERTER FOR ACTIVE DISTORTION COMPENSATION AND POWER FACTOR CORRECTION
}

\author{
Simon D. ROUND and Richard M. DUKE \\ Department of Electrical \& Electronic Engineering \\ University of Canterbury, Christchurch, New Zealand
}

\begin{abstract}
A technique of achieving both active current distortion compensation and power factor correction is described. A signal corresponding to the phase corrected distortion component of load current is amplified by a high frequency asynchronous switching inverter for addition back to the supply current to cancel the load current distortion and to provide power factor correction. Current distortion compensation and power factor correction can be provided either separately or combined to provide total compensation. Experimental results, illustrating the system's ability to reduce the current harmonic distortion components and provide power factor correction, are presented. The design of an intelligent controller, using a TMS320C30 digital signal processor, for the compensation system is also discussed.
\end{abstract}

\section{Introduction}

The detrimental effects of load current distortion and poor power factor can be solved by removing the load current distortion components and leaving a "clean" power factor corrected sinusoid. Commonly this is achieved by the addition of passive filters at the point of supply and shunt capacitors connected across the load.

Passive filters are however, non-selective, so they not only filter out the unwanted harmonic components from the supply they are installed on, but they also serve as a "sink" for the distortion components produced by other consumers in the vicinity. They are also generally tuned to remove specific frequency components and are therefore not a completely satisfactory solution when the harmonic composition of the distorted waveform changes. An active filter however is not tuned to remove specific frequency components and can provide varying amounts of distortion compensation to cater for load changes.

Banks of power factor correction capacitors cannot correct power factor for varying loads, they can provide total correction only for a selection of loads. Active power factor correction techniques can, on the other hand, provide total correction for a continuously variable range of loads.

An active compensation system has been developed which can directly cancel any unwanted current distortion and at the same time provide power factor correction. The system is based on current reinjection into the power supply using a high frequency controlled current inverter configuration as developed for use in $\mathrm{AC}$ variable speed drives ${ }^{1-2}$.

\section{The Active Compensation System}

The active compensation system, which employs a high frequency time based hysteresis controlled current inverter, is shown in Fig. 1.

A sinusoid corresponding to the power factor corrected fundamental component of load current $\left(\mathrm{I}_{\mathrm{L}}\right)$, which may be comprised of fundamental and distortion components, is extracted by the Signal Processing Unit (SPU) $)^{34}$. Digital circuitry is used for synthetically generating the fundamental component of $I_{L}$ from a look-up EPROM. Subtraction of this power factor corrected fundamental component from $I_{L}$ leaves the distortion component of load current, which is then amplified by the controlled current inverter to form the compensating current $\left(\mathrm{I}_{\mathrm{C}}\right) . \mathrm{I}_{\mathrm{C}}$ is then added to the supply via a reinjection transformer. This results in distortion current cancellation and a nearly sinusoidal power factor corrected supply current $\left(\mathrm{I}_{\mathrm{S}}\right)$.

\section{Experimental Verification}

The active compensation technique has been evaluated using a single phase prototype circuit that provides up to $2 \mathrm{kVA}$ of distortion compensation (Fig. 2). The non-linear 


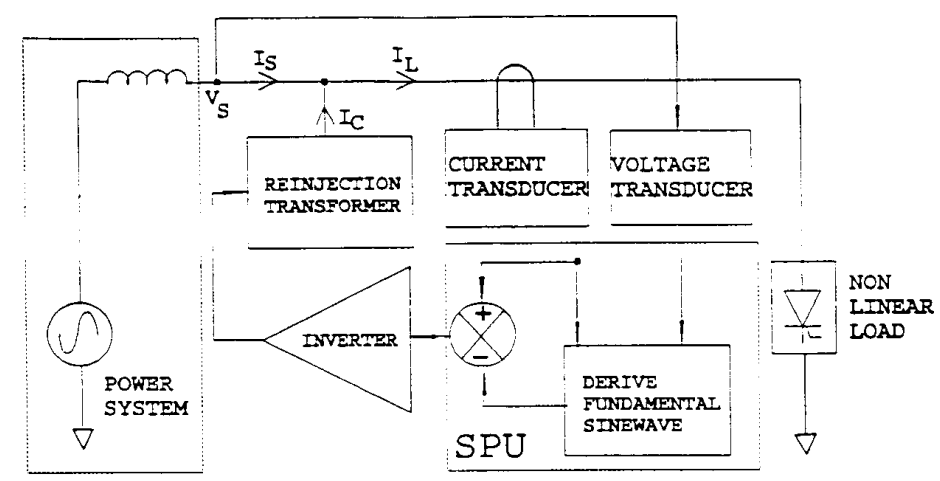

Fig. 1 Active compensation system

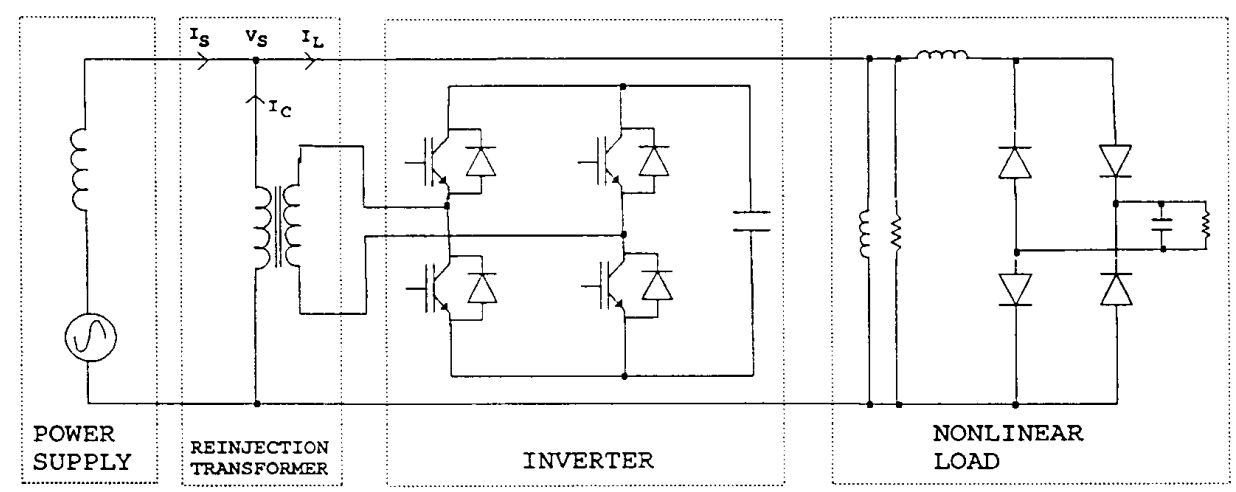

Fig. 2 Prototype circuit

load consists primarily of a resistive-inductive base load combined with a typical single phase rectifier load. The resistive and inductive components of the base load are rated $500 \mathrm{~W}$ and $900 \mathrm{VAr}$ respectively and draw a combined current of $6 \mathrm{~A}$ rms. The rectifier load is rated $300 \mathrm{~W}$ and delivers $1 \mathrm{~A}$ DC. The power supply for both the active distortion compensation system and the non-linear load is provided at $240 \mathrm{~V}$ from a $50 \mathrm{~Hz}$ system. The switching inverter, which uses IGBTs, is interfaced to the power supply via a reinjection transformer of ratio $2: 1$. The inverter output current is sensed using a resistive shunt and the comparison of inverter output current and compensating current signal takes place referenced to the inverter output. The inverter control logic activates either of the two diagonal pairs of transistors, to apply a positive or negative voltage across the liansformer connected load. Compensating current then ramps up or down at a rate determined by the time constant of the reinjection transformer.

\section{Switching strategy}

With reference to the transistor switching diagram
(Fig.3), the transistor switching instants are determined in the following way: Assuming the actual inverter output current is greater than the compensating current signal (point $\mathrm{A}$ ), a negative voltage is applied across the transformer connected load and the actual inverter current ramps down. This ramp down of inverter current continues for $t \mu \mathrm{s}$ after crossing the compensating current signal at point $\mathrm{B}$. At point $\mathrm{C}$ the

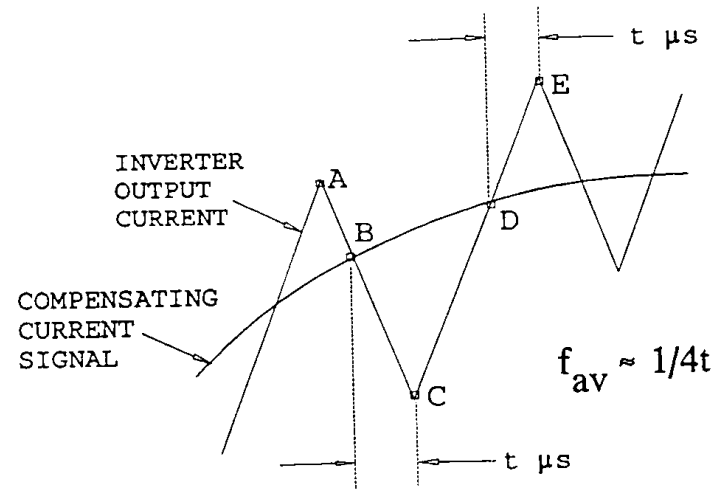

Fig. 3 Transistor switching diagram 
alternate pair of diagonal transistors are activated and a positive voltage is applied across the transformer connected load. The actual inverter current now ramps up, continuing past point $D$ for $t \mu \mathrm{s}$. This hysteresis time delay of $t \mu \mathrm{s}$ sets the average switching frequency $\left(f_{\mathrm{dv}} \approx 1 / 4 t\right)$ of the inverter. Clearly the actual switching frequency may vary over a wide range when the hysteresis time delay is fixed and the slope of the required compensating current varies. Hence the range of transistor switching frequencies, which are asynchronous, are determined by the required compensating current waveform.

\section{Computer Simulation}

Since the switching operations of the asynchronously controlled current inverter cannot be prespecified a dynamic state-space modelling technique is necessary. Such a statespace formulation has been developed and verified ${ }^{4}$ for analysis of the active compensation system. Simulations described in this paper have been carried out using the MATLAB simulation and analysis software package.

\section{Performance}

The performance of the active compensation system has been assessed initially from the point of view of providing supply current distortion compensation only. Active power factor correction has then been combined with distortion compensation to provide total compensation, producing a unity power factor sinusoidal line current at the point of supply.

\section{Distortion Compensation}

The distortion of the load current $\mathrm{I}_{\mathrm{L}}$ is evident from Fig. 4(a) and the measured Total Harmonic Distortion (THD), taking into account frequencies up to $1050 \mathrm{~Hz}$, is $24.8 \%$. The compensating current $\mathrm{I}_{\mathrm{C}}$ is shown in Fig. 4(b) and the compensated sinusoidal supply current $I_{S}$ (THD 1.53\%) and supply voltage $V_{S}$ are shown in Fig. $4(c)$. Switching frequency variability is apparent from the trace of $I_{C}$ in Fig. 4(b) and the distribution of these switching frequencies is shown in the computer simulation results of Fig. 4(d). The range of switching frequencies extends from $7 \mathrm{kHz}$ to $28 \mathrm{kHz}$ with an average switching frequency of $21 \mathrm{kHz}$.

\section{Total Compensation}

The load current $I_{L}$ shown in Fig. 5(a) is identical with that shown in Fig. 4(a). However now that power factor
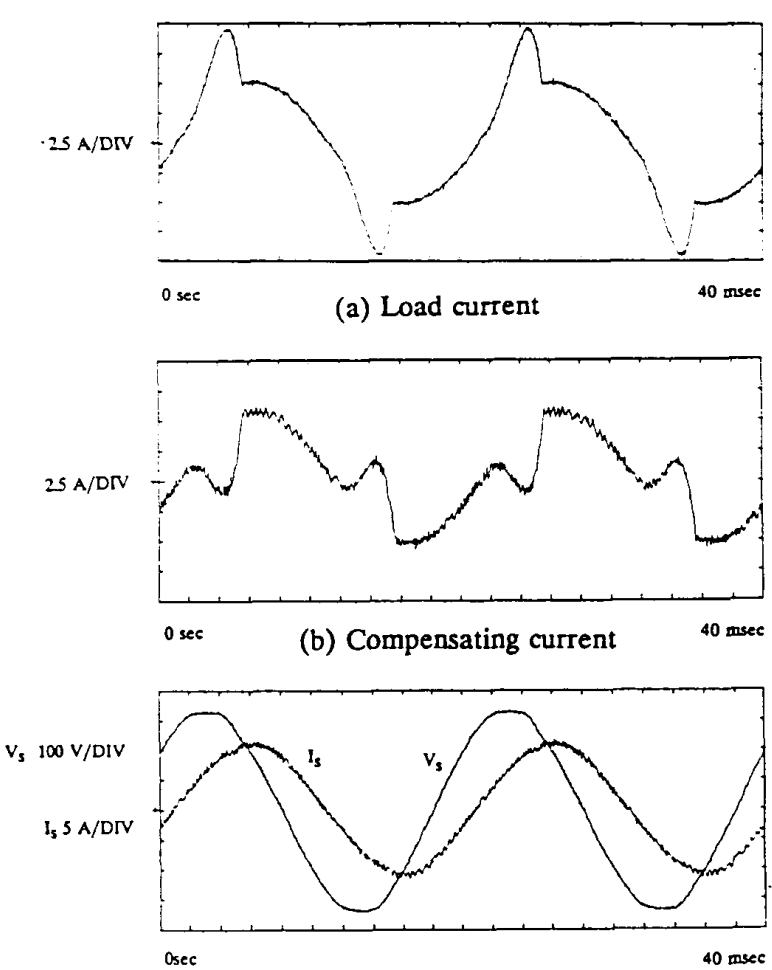

(c) Supply voltage and compensated supply current

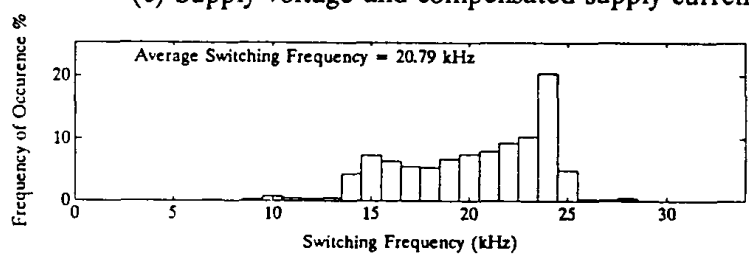

(d) Switching frequency distribution

Fig. 4 Distortion compensation

correction has been included along with distortion compensation, the compensating current $I_{C}$, shown in Fig. 5(b), is quite different. The compensated sinusoidal supply current $I_{S}$ and supply voltage $V_{S}$ are shown in Fig. $5(c)$. In addition to the reduction in THD, from $24.8 \%$ in Fig. 5(a) to $4.3 \%$ in Fig. $5(\mathrm{c}), I_{S}$ has been phase shifted by $55^{\circ}$ and is now in phase with $V_{s}$. The actual phase displacement between $V_{S}$ and $I_{S}$ being less than $1^{\circ}$.

Figs. 5(d) and (e) show the spectra of $I_{L}$ and $I_{S}$ respectively. With total compensation the 3 rd harmonic $(150 \mathrm{~Hz})$ has been reduced by $15 \mathrm{dBV}$ and is now $28 \mathrm{dBV}$ below the fundamental (3.98\% of the fundamental), while the Sth harmonic $(250 \mathrm{~Hz})$ has been reduced by $22 \mathrm{dBV}$ and is now $40 \mathrm{dBV}$ below the fundamental $(1.0 \%$ of the fundamental). 

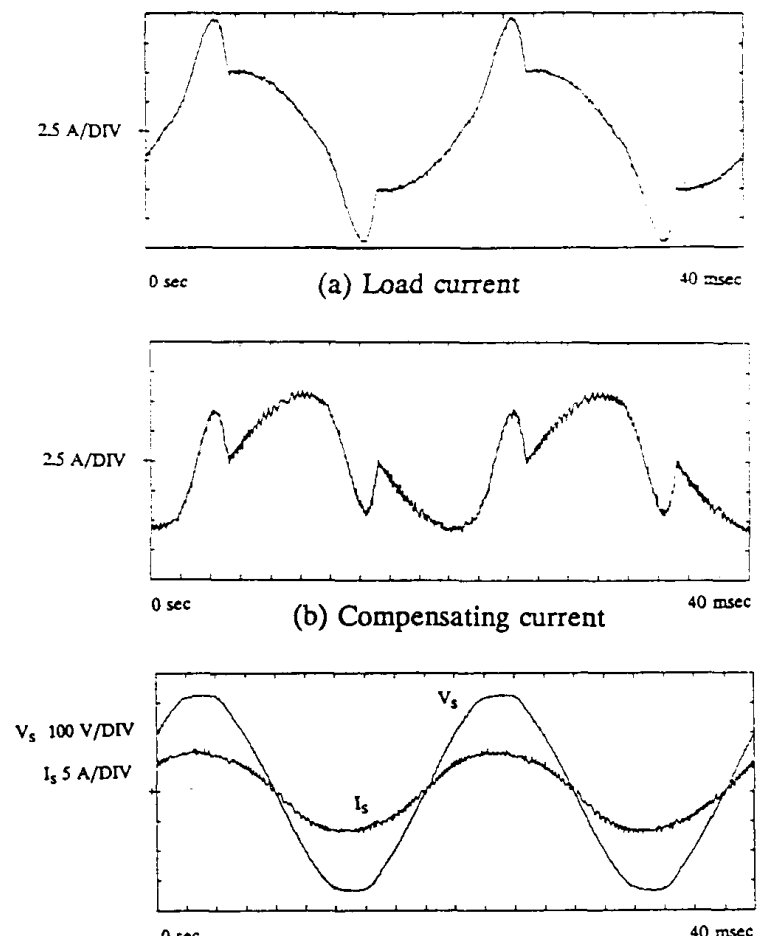

(c) Supply voltage and compensated supply current

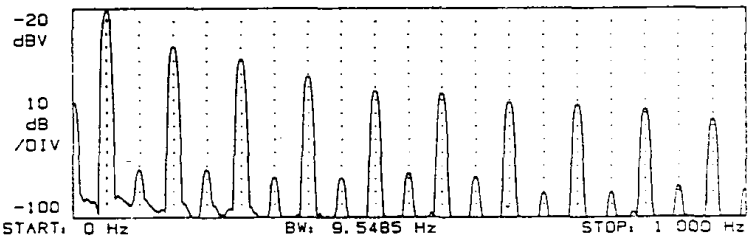

(d) Load current spectrum

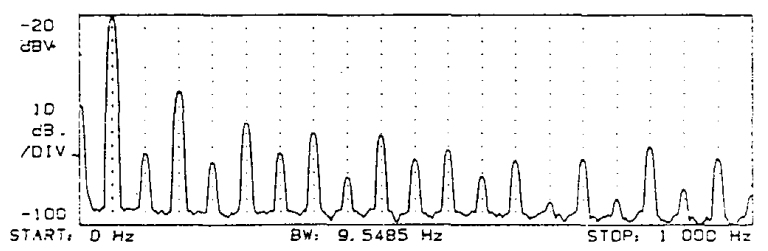

(e) Compensated supply current spectrum

Fig. 5 Total compensation

\section{Operating Efficiency}

Operating efficiency of the active compensation system is based on the real power flow in the system and is determined from the fundamental current that is in phase with the supply voltage. Assuming that the compensation system is supplied from a strong ac system, the real power flow is the product of the voltage and in phase fundamental current component. The efficiency $(\zeta)$ of the compensation system can then be defined by

\section{$\zeta=\frac{P_{\text {load curzent }}}{P_{\text {aupply cur ont with componsation }}}$}

Fig 6(a) shows the measured efficiency of the active compensation system with different average switching frequencies and DC bus voltages when providing total compensation. The efficiency improves with low bus voltages because the rate of rise of current has decreased. The switching frequency profile now contains a greater proportion of lower frequency components. Hence the switching losses decrease because the inverter is switching at a lower average frequency and a lower bus voltage is applied across the semiconductors. As the bus voltage is increased, for the same average switching frequency, the semiconductor switching losses increase significantly and efficiency decreases. At low frequencies (less than $5 \mathrm{kHz}$ ) the hysteresis band is much larger, thus the current excursions are increased and the conduction losses of the system are dominant. At high switching frequencies (greater than $20 \mathrm{kHz}$ ) the switching losses of the system become more dominant and thus the efficiency tends to fall. The most efficient average switching frequency for this particular non-linear load is in the range $4-14 \mathrm{kHz}$. To establish an appropriate bus voltage operating level the THD performance of the compensation system must also be considered.

The level of supply current THD at various bus voltages and average switching frequencies is shown in Fig. 6(b). At low bus voltages the THD performance is poor because the DC bus has insufficient stored energy to reinject enough compensating current for complete harmonic cancellation and power factor correction. By increasing the bus voltage the system can better compensate for the harmonic distortion and lagging power factor, however, the incremental increase in THD at higher bus voltages is small. The other factor that can alter the THD performance is the average switching frequency. At low switching frequencies (less than $9 \mathrm{kHz}$ ) the THD can be up to three times larger than that achieved at higher frequencies. The reason for this trend is that at low frequencies the hysteresis band is much larger and thus the harmonic compensation is less then ideal. As the frequency increases the inverter output current can follow the required compensating current signal far more closely and thus the harmonic content can be further reduced. 
(a)

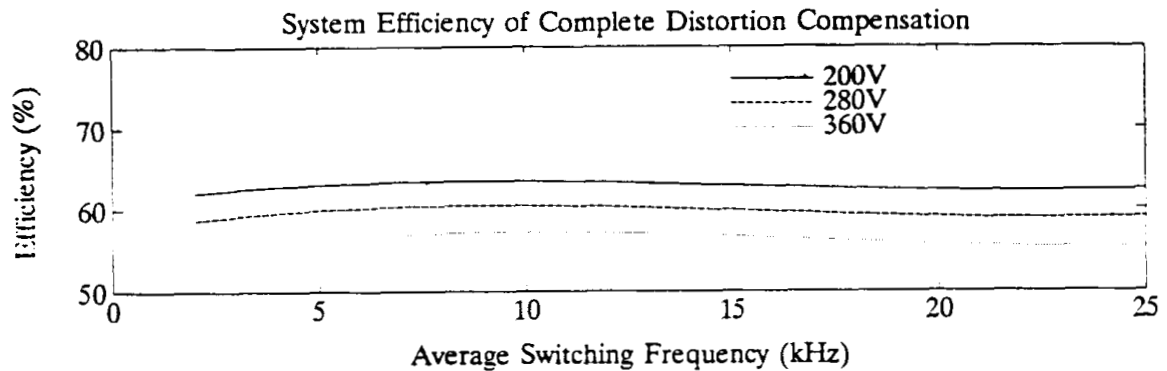

(b)

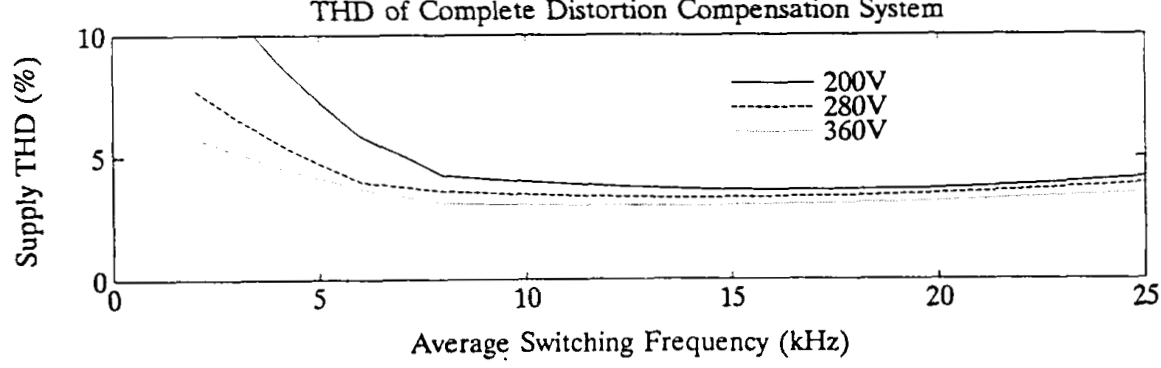

Fig. 6 Performance characteristics

\section{Intelligent Control}

By changing the average switching frequency and the DC bus voltage of the inverter an optimum operating point, which achieves low supply current distortion at maximum efficiency, can be reached. However with different types of loads, this optimum operating point changes. Therefore an intelligent controller, which monitors the efficiency and distortion compensation performance, and alters the operating point to achieve optimal performance, is proposed.

The system has been designed to be accurate within one degree of the fundamental $(50 \mathrm{~Hz})$. Thus the controller has to measure $I_{L}$, subtract a sinusoid and send the resulting distortion signal to the inverter within $55 \mu \mathrm{s}$. Therefore a sampling frequency of $25 \mathrm{kHz}$ is necessary and a high speed controller is required. To achieve real time operation, a TMS320C30 Digital Signal Processor (DSP) has been chosen as the controller. The hardware configuration of the DSP distortion compensation system is shown in Figure 7.

The load current is sampled by a 14 bit ADC at approximately $25.6 \mathrm{kHz}$. This sampling frequency is varied so that there are exactly 512 samples in each fundamental period. The variation in sample rate is necessary since the fundamental frequency is not constant (variations of $\pm 1 \%$ may occur). The fundamental zero crossing is used to

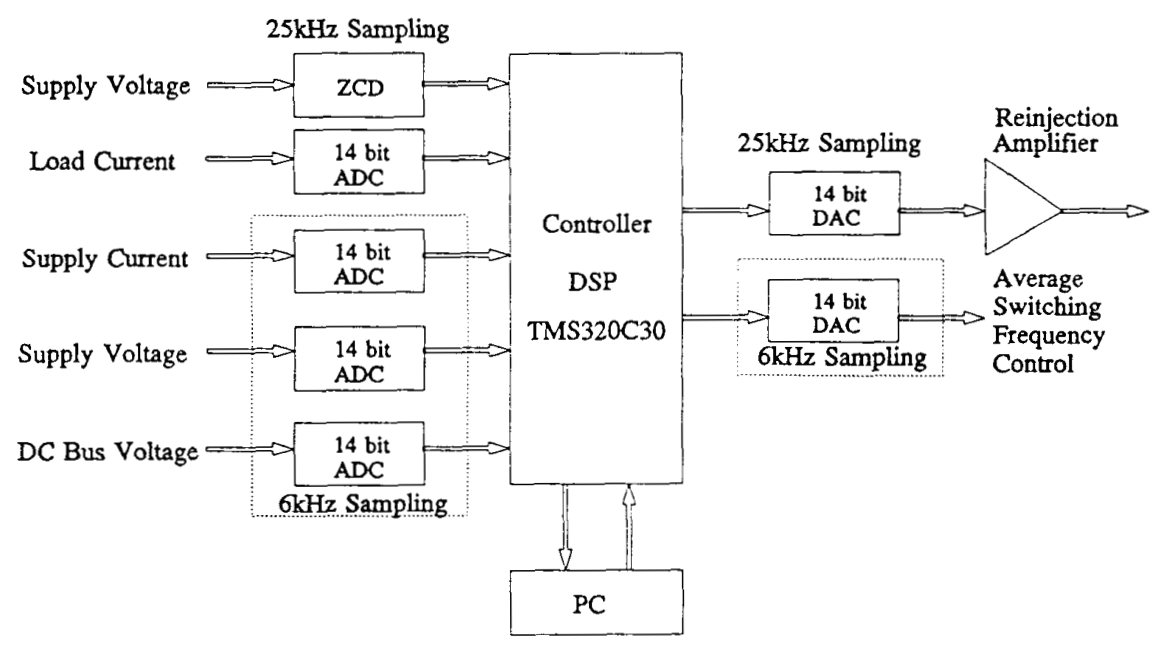

Fig. 7 Intelligent controller

\section{ECON'91}


synchronise the sampling frequency. Each load current sample is sent to the DSP, where the instantaneous phase corrected sinusoidal value is subtracted, leaving the distortion signal. The magnitude of the sinusoid is controlled by the voltage level on the DC bus capacitors and the instantaneous load current level, the resulting distortion signal is fed via a 14 bit $\mathrm{DAC}$ to the inverter where it is amplified and reinjected into the supply.

Using a second 14 bit DAC the average switching frequency is controlled in the range from 1 to $40 \mathrm{kHz}$. Three 14 bit $\mathrm{ADCs}$, sampling at a quarter of the sampling frequency of the load current, are used to monitor the supply current and voltage and the DC bus voltage. Using the supply voltage information the system can perform power factor correction and together with the load and supply current information calculate the efficiency of the system.

\section{Conclusion}

It has been demonstrated that an active compensation system using a high frequency asynchronously controlled current inverter is effective in phase shifting and reducing the harmonic content of distorted current waveforms. The novel use of a digitally generated sinewave in the SPU has provided an effective means for deriving the required compensating signal for any arbitrary load current waveform. There is an optimum operating range for the active compensation system which is determined by the choice of average switching frequency (hysteresis time) and DC bus voltage of the inverter. The design of an intelligent controller, which monitors the efficiency and distortion compensation performance and alters the operating point to achieve optimal performance of the active compensation system, has also been outlined.

\section{References}

[1] Brod D.M. and Novotny D.W.: "Current control of VSI-PWM inverters", IEEE Trans. 1985, IA-21, pp. $562-570$.

[2] Penny J.R.: "Modern controlled-current AC drives", Proc. of a seminar "Power Electronics", Annual Conf. of IPENZ, 1987, Vol. 13, pp. 19 - 26.

[3] Henderson K.C.: "The development of an active harmonic filter for power systems", Proc. IPENZ Annual Conf., 1990, Vol.II, pp.129 - 138.

[4] Duke R.M., Round S.D. and Henderson K.C.: "An active filter for current distortion compensation in power systems", Proc. 4th Conf. on Harmonics in Power Systems, Budapest, Hungary. Oct.4-6 1990, pp.367 - 73. 